\title{
Recent advances in airway management in children
}

\section{Francis Veyckemans}

\author{
Address: Department of Anesthesiology, Catholic University of Louvain and Cliniques universitaires St Luc, Avenue Hippocrate, 10-1821, \\ B-1200 Brussels, Belgium \\ Email: francis.veyckemans@uclouvain.be
}

FI000 Medicine Reports 2009, I:72 (doi:I0.34I0/MI-72)

The electronic version of this article is the complete one and can be found at: http://FI000.com/Reports/Medicine/content/I/72

\begin{abstract}
Recent anatomic findings, technological progress, and both in vitro and in vivo studies of the pressure generated in the cuff of endotracheal tubes and supraglottic airways should lead to modification of the way we control the pediatric upper airway.
\end{abstract}

\section{Introduction and context}

The use of uncuffed endotracheal tubes in children up to 8 years of age is partly based on the classical description of the infant's and child's larynx: funnel-shaped with the apex at the level of the cricoid and a round section at the level of the cricoid cartilage. This concept has been challenged by recent in vivo studies. The increased use of polyvinyl chloride (PVC) endotracheal tubes with highvolume low-pressure cuffs in pediatric patients does not increase the incidence of post-operative stridor compared with uncuffed tubes, but is this outcome a reliable sign of airway injury? The cuff of supraglottic airways is usually inflated up to the point when satisfactory leak pressure is achieved, although monitoring intracuff pressure is also necessary. Fiberoptic bronchoscopy is the gold standard in case of difficult intubation, however, the availability of new videolaryngoscopes and optic laryngoscopes could modify our practice and improve the difficult airway algorithms.

\section{Recent advances Upper airway anatomy}

The classical description of the infant's and child's larynx is based on cadaver studies. Two in vivo studies were published recently: one using magnetic resonance imaging in sedated children [1], the other videobronchoscopy in paralyzed children [2]. According to these studies, the glottis rather than the cricoid is the narrowest part of the pediatric airway, which is also more cylindrical than funnel-shaped. Moreover, the cricoid section is ellipsoid, not round. However, the vocal cords are mobile and can thus enlarge the glottic opening when a tracheal tube is inserted, whereas the diameter of the cricoid ring is fixed; this explains why postintubation injuries can be observed at both the vocal cords and the subglottic level.

\section{Cuffed endotracheal tubes}

These findings and the design of pediatric-sized tracheal tubes with high-volume low-pressure cuffs has made the use of cuffed tracheal tubes more and more popular in pediatric anesthesia and intensive care. A few epidemiological studies have shown that the incidence of postintubation stridor is similar when a cuffed or uncuffed tube is used $[3,4]$. However, post-intubation stridor is not a good indicator of the presence and severity of airway injury: stridor can indeed be a sign of early benign edema or severe obstruction but large areas of mucosal necrosis do not cause stridor until later when scarring narrows the airway by more than 50\%. Endoscopic examination of the airway after extubation is the best way to assess post-intubation airway injury and its causes [5]. Moreover, an air leak should be present at $20 \mathrm{cmH}_{2} \mathrm{O}$ around the tube with its cuff uninflated. Lastly, the cuff should be carefully positioned below the cricoid area because most endoscopy-proven post-intubation laryngeal injuries are caused by folds of a deflated cuff and malposition of the cuff $[5,6]$. 
In vitro studies using a tracheal model have demonstrated that, regardless of its initial value (low or high) when tested in an unrestricted environment, compliance of the cuff decreases considerably when placed in the model. More importantly, this study also showed that tracheal wall pressure increased with cuff pressure if the cuff was sufficiently inflated to drape the inner tracheal wall. Accordingly, even the use of high-volume low-pressure cuffs does not avoid high intratracheal pressures being generated; cuff pressure monitoring (and limitation to $20 \mathrm{cmH}_{2} \mathrm{O}$ ) is therefore mandatory [7].

\section{Supraglottic airways}

In vitro studies have shown that injecting the maximum recommended cuff filling volumes in pediatric laryngeal mask airways (LMAs) results in hyperinflation in almost all models tested [8]. This has been confirmed by measuring the cuff pressure-volume relationship in vivo: injecting approximately $50 \%$ of the maximum volume recommended resulted in intracuff pressures in excess of $60 \mathrm{cmH}_{2} \mathrm{O}$ [9]. As a higher intracuff pressure can result in pharyngeal morbidity and increased leakage of air around the LMA, monitoring intracuff pressure is now strongly recommended [10].

\section{Videolaryngoscopes}

Both Miller and Macintosh blades equipped with an external fiber optic bundle or in which a fiber optic lens is integrated and coupled with a camera through the handle are available. They are useful for teaching direct laryngoscopy and when external laryngeal manipulation is necessary for intubation (the helper and the operator see the same picture). A better view of the glottis is obtained but intubation is not always easier because some training is necessary to learn how to use a flat twodimensional screen instead of the real three-dimensional image to guide the tube and because less force is applied on the intraoral structures than during direct laryngoscopy $[11,12]$.

The Glidescope ${ }^{\circledR}$ Cobalt is an image tube enclosed in a reusable handle with a single-use blade bent at $60^{\circ}$. It provides a view of the laryngeal structure on a separate screen. The glottic view is easy and excellent, but it is often not easy to introduce the tube into the glottic opening; for oral intubation, using either the accompanying stylet or a standard stylet to bend the endotracheal tube into a hockey stick shape makes the manipulation of the endotracheal tube toward the glottis easier. Apparently, bending the stylet at $90^{\circ}$ rather than at $60^{\circ}$ makes oral intubation more often successful at the first attempt [13]. This device should be introduced under direct vision in the middle of the tongue with the head in the neutral position. One should then look at the monitor to obtain the best view of the epiglottis and glottic opening; the blade is generally used like a Macintosh blade, that is, without lifting the epiglottis. The tube and its stylet are then carefully introduced from the right corner of the mouth under direct vision up to near the tip of the blade to avoid any damage to intraoral structures [14].

The Airtraq ${ }^{\circledR}$ is a single use indirect laryngoscope in which the exaggerated curvature of the blade and the configuration of the optical components provide a view of the glottis without the need to align the oral, pharyngeal, and tracheal axes. It should be introduced under direct vision in the middle of the tongue with the head in the neutral position. When the monitor gives the best view of the epiglottis, the tip should be placed in the vallecula. The handle should then be lifted up vertically to lift the epiglottis and expose the glottic opening. The latter should be aligned with the center of the visual field because the right-sided groove is designed to direct the tip of the tube towards the center of the visual field. The tip of the endotracheal tube is then guided into the glottic opening under direct visual control. The Airtraq ${ }^{\circledR}$ improves visualization of the glottis and facilitates both normal and difficult intubation in adults while reducing the hemodynamic response to laryngoscopy. Only a few case reports of successful intubation in difficult pediatric intubation cases have been published so far [15].

These devices are less expensive than a fiber optic bronchoscope but also need some training in children with a normal airway before being used for difficult intubation, however, their learning curve is probably steeper.

\section{Implications for clinical practice}

Monitoring cuff pressure in endotracheal tubes and in supraglottic airways is recommended to lessen the risk of mucosal damage. Regarding cuffed endotracheal tubes, they should be used carefully: a leak should be present at $20 \mathrm{cmH}_{2} \mathrm{O}$ before inflating the cuff, which must be placed below the cricoid area. Moreover, post-extubation endoscopic examination studies are necessary to assess their safety. Lastly, the new intubating devices should be evaluated in difficult intubation cases.

\section{Abbreviations}

LMA, laryngeal mask airway; PVC, polyvinyl chloride.

\section{Competing interests}

The author participated in a multicentric clinical study evaluating the safety of cuffed versus uncuffed tracheal tubes in children less than 5 years of age under the 
direction of Professor M Weiss and AC Gerber (Zurich). Professor M Weiss and AC Gerber developed a commercially available pediatric cuffed endotracheal tube (Microcuff ${ }^{\circledR}$ ).

\section{References}

I. Litman RS, Weissend EE, Shibata D, Westesson PL: Developmental changes of laryngeal dimensions in unparalyzed sedated children. Anesthesiology 2003, 98:4I-5.

2. Dalal PG, Murray D, Messner AH, Feng A, McAllister J, Molter D: Pediatric laryngeal dimensions: an age-based analysis. Anesth Analg 2009, 108:1475-9.

Changes Clinical Practice

FI000 Factor 6.6 Must Read

Evaluated by Victor Baum 12 May 2009, Anne Lynn OI Jul 2009. Jerome Parness 27 Jul 2009

3. Newth CJL, Rachman B, Patel N, Hammer J: The use of cuffed versus uncuffed endotracheal tubes in pediatric intensive care. J Pediatr 2004, 144:333-7.

4. Khine HH, Corddry DH, Kettrick RG, Martin TM, McCloskey J, Rose JB, Theroux MC, Zagnoev M: Comparison of cuffed and uncuffed endotracheal tubes in young children during general anesthesia. Anesthesiology 1997, 86:627-3I.

5. Holzki J, Laschat M, Puder C: Stridor is not a scientifically valid outcome measure for assessing airway injury. Paediatr Anaesth 2009, I9(Suppl I): I80-97.

6. Dillier CM, Trachsel D, Baulig W, Gysin C, Gerber AC, Weiss M: Laryngeal damage due to an unexpectedly large and inappropriately designed cuffed pediatric tracheal tube in a 13-month-old child. Can J Anaesth 2004, 5 1:72-5.

7. Bernet $V$, Dullenkopf $A$, Cannizzaro V, Stutz K, Weiss M: An in vitro study of the compliance of paediatric tracheal tubes and tracheal wall pressure. Anaesthesia 2006, 61:978-83.
8. Maino P, Dullekopf A, Keller C, Bernet-Buettiker V, Weiss M: Cuff filling volumes and pressures in pediatric laryngeal mask airways. Pediatr Anesth 2006, 16:25-30.

9. Wallace Cl, Chambers NA, Erb TO, von Ungern-Sternberg BS: Pressure volume curves of paediatric laryngeal mask airways. Anaesthesia 2009, 64:527-31.

Changes Clinical Practice

FI000 Factor 6.0 Must Read

Evaluated by Francis Veyckemans 13 May 2009

10. Licina A, Chambers NA, Hullett B, Erb TO, von Ungern-Sternberg BS: Lower cuff pressures improve the seal of pediatric laryngeal mask airways. Pediatr Anesth 2008, 18:952-6.

FI000 Factor 6.0 Must Read Evaluated by Linda Mason 13 Oct 2008

II. Macnair D, Baraclough D, Wilson G, Bloch M, Engelhardt T: Pediatric airway management: comparing the Berci-Kaplan laryngoscope with direct laryngoscopy. Pediatr Anesth 2009, 19:577-80

12. Fiadjoe JE, Stricker PA, Hackell RS, Salam A, Gurnaney H, Rehmann MA, Litman RS: The efficacy of the Storz Miller I video laryngoscope in a simulated infant difficult intubation. Anesth Analg 2009, 108:1783-6.

13. Redel A, Karademir F, Schlitterlau A, Frommer M, Scholtz LU, Kranke $P$, Kehl F, Roewer N, Lange M: Validation of the GlideScope video laryngoscope in pediatric patients. Paediatr Anaesth 2009, 19:667-71.

14. Vincent RD, Wimberly MP, Brockwell RC, Magnuson JS: Soft palate perforation during orotracheal intubation facilitated by the Glidescope videolaryngoscope. J Clin Anesth 2007, 19:6 I9-21.

15. Vlatten A, Soder C: Airtraq optical laryngoscope intubation in a 5-month-old infant with a difficult airway because of Robin sequence. Pediatr Anesth 2009, 19:699-700. 